\title{
Correction to: Palbociclib use with grade 3 neutropenia in hormone receptor-positive metastatic breast cancer
}

\author{
Ahrong Ham ${ }^{1}$ - Min Hwan Kim ${ }^{1}$. Gun Min Kim ${ }^{1}$. Jee Hung Kim ${ }^{2}$. Jee Ye Kim ${ }^{3}$. Hyung Seok Park ${ }^{3}$. Seho Park ${ }^{3}$. \\ Young Up $\mathrm{Cho}^{3}$ - Byeong Woo Park ${ }^{3}$. Seung II Kim ${ }^{3}$. Joohyuk Sohn ${ }^{1}$ (D)
}

Published online: 14 July 2020

○) Springer Science+Business Media, LLC, part of Springer Nature 2020

\section{Correction to: Breast Cancer Research and Treatment https://doi.org/10.1007/s10549-020-05750-y}

In the original publication of the article, under the Results section, subheading "Patient survival", the second sentence that reads as "The 6-month PFS was $92.4 \%, 81.8 \%$, and 93.3\% and the 1-year PFS was $72.0 \%, 88.9 \%$, and $78.9 \%$ in Groups 1-3, respectively." should read as "The 6-month PFS was $82.8 \%, 75.0 \%$, and $68.0 \%$ and the 1 -year PFS was $77.0 \%, 62.0 \%$, and $63.8 \%$ in Groups $1-3$, respectively.".

Publisher's Note Springer Nature remains neutral with regard to jurisdictional claims in published maps and institutional affiliations.

The original article can be found online at https://doi.org/10.1007/ s10549-020-05750-y.

Joohyuk Sohn

oncosohn@yuhs.ac

1 Division of Medical Oncology, Department of Internal Medicine, Yonsei Cancer Center, Yonsei University College of Medicine, 50-1 Yonsei-ro, Seodaemun-gu, Seoul 03722, Republic of Korea

2 Division of Medical Oncology, Department of Internal Medicine, Gangnam Severance Hospital, Yonsei University College of Medicine, Seoul, Republic of Korea

3 Division of Breast Surgery, Department of Surgery, Yonsei Cancer Center, Yonsei University College of Medicine, Seoul, Republic of Korea 